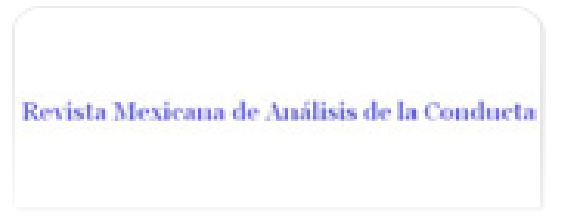

Revista Mexicana de Análisis de la Conducta ISSN: 0185-4534

editora@rmac-mx.org

Sociedad Mexicana de Análisis de la Conducta México

RUVALCABA PALACIOS, GERARDO; DOMÍNGUEZ TREJO, BEMJAMÍN Efectos psicológicos y físicos de la modulación autonómica en el dolor miofascial: un estudio aleatorizado

Revista Mexicana de Análisis de la Conducta, vol. 37, núm. 2, 2011, pp. 99-115

Sociedad Mexicana de Análisis de la Conducta

Guadalajara, México

Disponible en: http://www.redalyc.org/articulo.oa?id=59319255007

- Cómo citar el artículo

- Número completo

- Más información del artículo

Página de la revista en redalyc.org

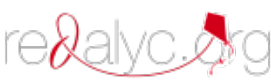

Sistema de Información Científica

Red de Revistas Científicas de América Latina, el Caribe, España y Portugal Proyecto académico sin fines de lucro, desarrollado bajo la iniciativa de acceso abierto 
REVISTA MEXICANA DE ANÁLISIS DE LA CONDUCTA MEXICAN JOURNAL OF BEHAVIOR ANALYSIS

DOI: $10.5514 /$ rmac.v37.i2.26142
2011

$37,99-115$
NUM $2(8-11 / 11-11)$

\title{
EFECTOS PSICOLÓGICOS Y FÍSICOS DE LA MODULACIÓN AUTONÓMICA EN EL DOLOR MIOFASCIAL: UN ESTUDIO ALEATORIZADO
}

\author{
PSYCHOLOGICAL AND PHYSICAL EFFECTS OF AUTONOMIC MODULATION \\ ON MYOFASCIAL PAIN: A RANDOMIZED CONTROLLED TRIAL
}

\author{
GERARDO RUVALCABA PALACIOS ${ }^{1}$ Y BENJAMÍN DOMÍNGUEZ TREJO \\ ${ }^{1}$ Departamento de Enfermería y Obstetricia, Universidad de Guanajuato, \\ campus Irapuato-Salamanca, Guanajuato, México. \\ ${ }^{2}$ Facultad de Psicología, Universidad Nacional Autónoma de México, Ciudad de México, D.F., México
}

Resumen

En la Teoría Polivagal (Porges, 2001) se establece que el desarrollo de los síntomas psicológicos y físicos de los síndromes crónicos está asociado con una elevada actividad simpática y que la modificación de ésta podría disminuirlos. En el presente estudio se evaluaron dichos supuestos en pacientes con dolor miofascial. 30 mujeres fueron asignadas aleatoriamente a un grupo experimental ("entrenamiento") o control ("platicas para la salud"). Antes y después de la intervención se midió la actividad autonómica (Temperatura Periférica y Electromiografía de Superficie), el estado cognitivo-emocional (Inventario de ansiedad y Depresión de Beck) y, mediante escalas de calificación numérica, los síntomas diagnósticos del dolor miofascial (Banda tensa, nódulo blando y dolor a la dígito-presión) así como la intensidad percibida de dolor. Al final el grupo que recibió entrenamiento mostró decrementos en todas las variables mientras que el grupo control no. Los resultados son congruentes con lo propuesto en la Teoría Polivagal por lo que es posible que el desarrollo y

\footnotetext{
Dirigir la correspondencia a: Gerardo Ruvalcaba Palacios, Departamento de Enfermería y Obstetricia, Manuel Mendivil S/N, Colonia Deportiva, Irapuato, Guanajuato, Teléfono (462) 621900 ext 11262 y 11270, e-mail: drruvalcaba@gmail.com. Se agradece el apoyo y la participación de Ma del Consuelo González Esquivel, Directora de Servicios Médicos y Ana Florencia Valtierra Bejines, Jefa del Departamento de Rehabilitación Física, ambas del Centro de Rehabilitación e Integración Social (CRIS) - DIF Aguascalientes y cuya disposición fue fundamental para el desarrollo del presente trabajo. La aportación de los autores fue como sigue: el planteamiento del problema, el diseño experimental, el análisis de datos, la integración de la discusión y la escritura del presente artículo es una labor conjunta de ambos autores. La recolección de datos y la escritura de la sección de resultados estuvieron a cargo del primer autor, figurando el segundo como revisor.
} 
Ruvalcaba \& Domínguez

permanencia de síndromes crónicos se relacione con el tipo de actividad nerviosaadaptativa de los pacientes. Se discuten las implicaciones de estos hallazgos para la medicina conductual.

Palabras clave: Teoría polivagal, actividad autonómica, dolor crónico, síndrome miofascial, retroalimentación biológica.

\section{Abstract}

According with Polyvagal Theory (Porges, 2001), development of the psychological and physical symptoms that distinguish chronic syndromes is related with an elevated sympathetic activity and, in the other hand, reductions in this activity could derive in symptoms decrease. In this study, aforementioned assumptions were tested in chronic myofascial pain patients. 30 female patients were randomly assigned to one of two groups: experimental ("training") or control ("health chats"). Before and after intervention autonomic activity was recorded (Peripheral Temperature and Surface Electromyography) as well as cognitive-emotional state (Beck's Anxiety and Depression Inventory), and through numerical rating scales, both myofascial-pain diagnostic symptoms (soft nodule, taut band and digit-pressure pain) and perceived pain intensity. Training group showed decrements in all variables under study while control group do not. Results are congruent with Polyvagal Theory and supports possibility for relationships between development and permanence of chronic syndromes and patient's nervous adaptive activity. Implications of these findings to behavioral medicine field are discussed.

Key words: Polyvagal theory, autonomic activity, chronic pain, myofascial syndrome, biofeedback.

\section{Introducción}

El síndrome doloroso miofascial (SDM) es la fuente más común de dolor músculo esquelético entre la población adulta a nivel mundial (Myburgh, Larsen \& Hartvigsen, 2008) y la forma más ordinaria de dolor regional en la clínica de primer nivel y en los centros especializados en la atención del dolor crónico (Staud, 2009). En México, cerca del $27.7 \%$ de las personas adultas sufren dolor de origen musculoesquelético siendo éste la principal causa de visitas al médico y de incapacidad laboral (Bistre, 2009)

Los criterios esenciales para diagnosticar el SDM incluyen la presencia de nódulos blandos e hipersensibles dentro de una banda muscular tensa (hace referencia a fibras musculares tirantes, al tacto se sienten como cuerdas), Ilamados Puntos Gatillo (PsG), los cuales pueden afectar a uno o más músculos y producir un patrón especifico de dolor referido (Myburgh, et al., 2008; Simons, 2004). Se forman por demandas musculares elevadas o alteradas, como mantener por tiempo prolongado una misma postura, soportar mucho peso, o realizar movimientos repetitivos. También a consecuencia de traumatismos (Huggenin, 2004). 
Son considerados como un estado patológico neuromuscular mantenido por una excesiva descarga simpática sobre la placa motora terminal, lo que resulta en la contracción sostenida de pequeños grupos de sarcómeras las cuales a su vez forman el "nódulo blando" y la "banda muscular tensa" (Simons, 2004). El empeoramiento de los síntomas y la permanencia de los PsG se atribuyen a una disfunción del eje hipotálamo-pituitaria-adrenal (HPA).

La persistente elevada actividad del eje HPA condiciona una reorganización neuroconductual que se relaciona con importantes alteraciones en diferentes órganos y sistemas como el óseo, muscular, gástrico y neuronal (Jänig, 2009; Sapolsky, 2001), que al final deriva en un estado de vulnerabilidad para la instalación de diferentes síndromes crónicos (Chrousos, 1992; Chrousos \& Gold, 1992; Melzack, 1999; Porges, 2001; Sapolsky, 2001, 2003; Schulkin, 2003).

En la Teoría Polivagal (Porges, 2001, 2007) se postula que los organismos enfrentan las demandas del medio utilizando una de tres estrategias neuroconductuales (ver tabla 1), las cuales han sido moldeadas por la evolución. Los organismos superiores, especialmente el hombre, utilizan preferentemente la estrategia más evolucionada llamada de "socialización", sin embargo si esta no le permite recuperar su equilibrio homeostático entonces tenderá a utilizar las anteriores, pasando por estados de estrés y ansiedad hasta llegar a la depresión y la extinción que ésta supone (Porges, 2007; Sapolsky, 2003).

Tabla 1.

Estrategias neuroconductuales adaptativas según la Teoría Polivagal

\begin{tabular}{clll}
\hline ESTRATEGIA & \multicolumn{1}{c}{ NEUROANATOMÍA } & \multicolumn{1}{c}{ FISIOLOGÍA } & CONDUCTAS \\
\hline INMOVILIZACIÓN & $\begin{array}{l}\text { Rama no mielinizada del vago, } \\
\text { emerge del núcleo dorsal motor, } \\
\text { inerva de manera importante al } \\
\text { corazón }\end{array}$ & $\begin{array}{l}\text { Inhibición de actividad simpática. } \\
\text { Su actividad prolongada es letal } \\
\text { para los mamíferos }\end{array}$ & $\begin{array}{l}\text { Fingirse muerto, } \\
\text { desmayarse, síncope, } \\
\text { depresión }\end{array}$ \\
MOVILIZACIÓN & $\begin{array}{l}\text { Basada en la actividad simpato } \\
\text { adrenal y del eje HPA }\end{array}$ & $\begin{array}{l}\text { Inhibición del vago no } \\
\text { mielinizado. Su actividad } \\
\text { prolongada se relaciona con } \\
\text { síndromes crónicos }\end{array}$ & $\begin{array}{l}\text { Estrés, ansiedad, } \\
\text { conductas de lucha } \\
\text { huida }\end{array}$ \\
SOCIALIZACIÓN & $\begin{array}{l}\text { Rama mielinizada del vago, } \\
\text { emerge del núcleo ambiguo }\end{array}$ & $\begin{array}{l}\text { Influye actividad cardiaca, de tal } \\
\text { manera que es posible transitar } \\
\text { rápidamente entre estados de } \\
\text { movilización y calma }\end{array}$ & $\begin{array}{l}\text { Socialización, } \\
\text { involucramiento con } \\
\text { el ambiente, } \\
\text { relajación. }\end{array}$ \\
\hline
\end{tabular}

Nota: HPA: Hipotálamo-Pituitaria-Adrenal

También se propone que para el ser humano es posible estimular el tránsito entre las estrategias menos evolucionadas (y que a largo plazo resultan dañinas para el organismo) a la más actual, saludable y promotora de una adecuada adaptación (Porges, 2001; Porges, Domínguez, Rangel \& Cruz, 2005). Estos supuestos se han aplicado con éxito en el tratamiento de trastornos relacionados con la socialización (por ejemplo autismo) y los ataques de pánico, aunque sus aplicaciones dentro 
del campo de la medicina conductual recién están comenzando a documentarse (Porges, et al. 2005).

Sin embargo y de acuerdo con lo anterior, es posible hipotetizar que tanto los síntomas físicos como psicológicos que presentan los pacientes con SDM dependen en gran medida de la organización neuroconductual con la que enfrentan su estado de dolor, y que por lo tanto, reducir dicha activación nerviosa se acompañará de una reducción en los síntomas físicos y psicológicos. Sin embargo estos supuestos no han sido corroborados.

El presente estudio se realizó con el objetivo de evaluar, mediante un estudio aleatorizado, si después de un entrenamiento para modificar la actividad autonómica los participantes mostraban decrementos en las características físicas de los PsG, los niveles de ansiedad y depresión y la intensidad percibida de dolor.

\section{Método}

\section{Participantes}

Treinta mujeres, entre 40 y 55 años de edad fueron seleccionadas de una población de pacientes con dolor muscular en espalda baja que recibía atención en un centro de rehabilitación física dependiente del gobierno del estado de Aguascalientes, México y fueron asignadas, mediante aleatorización simple a uno de dos grupos: el grupo A recibió entrenamiento para modificar su actividad autonómica utilizando retroalimentación biológica y el grupo B pláticas para la salud. Para ser incluidas deberían tener más de dos años de haber presentado el primer episodio de dolor, además y debido a que la literatura señala que el tipo de trabajo desempeñado influye en la presencia de determinados síntomas y síndromes de dolor muscular (Punnett \& Wegman, 2004), se controló la influencia de esta variable incluyendo sólo a quienes desempeñaban trabajos administrativos. No se incluyeron pacientes embarazadas, que padecieran enfermedad vascular, diabetes, cáncer o dolor neuropático, hernias discales, compresión nerviosa, o alguna alteración estructural de la columna. Tampoco si consumían de forma habitual tabaco, alcohol o alguna droga ilegal. Todas las participantes fueron informadas de la naturaleza de la intervención y de sus responsabilidades y derechos dentro de la misma; participaron voluntariamente y dieron su consentimiento informado, según los criterios éticos de la Declaración de Helsinki (1975).

\section{Reclutamiento de pacientes}

Mediante la revisión de expedientes se detectaron aquellas pacientes que cumplieran con los criterios de inclusión para luego ser invitadas a una entrevista de reclutamiento con el Investigador Principal (IP) la cual tenía por objeto verificar los criterios de inclusión-exclusión. A las seleccionadas se les pidió su consentimiento para ser incluidas en el proyecto, se les informó de la naturaleza de la intervención, las mediciones que se realizarían y los objetivos esperados. A todas se les comunicó que podían abandonar el estudio si así lo requerían. 
Conforme se iban seleccionando, las participantes eran asignadas por el IP al grupo A o B de acuerdo con una tabla de números aleatorios. Números pares se asignaban al primero y nones al segundo. Ningún otro colaborador conocía qué paciente pertenecía a cuál grupo.

Instrumentos y materiales

Inventario de Depresión de Beck, estandarizado para población mexicana. Consta de 21 grupos de cuatro oraciones, las cuales están numeradas del 0 al 4, el orden indica niveles de apreciaciones cognitivas respecto a la depresión. Las puntuaciones obtenidas por el paciente indican su nivel de depresión (Jurado, Villegas, Méndez, Rodríguez, Loperena \& Varela, 1998).

Inventario de Ansiedad de Beck. Estandarizado para población mexicana. Consiste en 21 reactivos que señalan diferentes síntomas físicos y cognitivos de ansiedad, el paciente los califica de acuerdo a una escala tipo likert con valencias de 1 "poco o nada" a 4 "severamente", según haya experimentado cada uno de ellos. La suma de las respuestas indica el nivel de ansiedad (Robles, Varela, Jurado \& Páez, 2001).

Escalas de calificación numérica. Consistió en una línea recta de $10 \mathrm{~cm}$ graduada desde 0 "ausencia de sensación o de la característica", hasta 10 "máxima presencia de la sensación o la característica". Para calificar la intensidad de los síntomas diagnósticos del SDM, la escala era puntuada por el especialista que realizaba la exploración. En el caso de la intensidad percibida de dolor, era el paciente quien la puntuaba (McQuay, 2005; Tait, 1999).

Perfil psicofisiológico del estrés. Es un formato en el que se encuentra espacio para cada una de las condiciones a registrar (Sentada ojos abiertos, Sentada ojos cerrados, Respuesta de estrés, Respuesta natural de relajación). A su vez, en cada condición hay espacio para registrar cada 15 segundos, durante 2 minutos la señal psicofisiológica elegida (Ruvalcaba \& Domínguez, 2009a).

Para registrar la actividad autonómica se utilizó un equipo de retroalimentación biológica "PROCOMP+" (Tought Technology, Co.), una computadora portátil y el software "Infinity" (Tought Technology, Co.).

Para limpiar el área de la piel en donde se colocarían los sensores se usó algodón y alcohol de curación. Para fijar los sensores, una cinta adhesiva hipoalergénica.

Diseño

Consistió en un diseño experimental pretest - postest con grupo de control (Kerlinger \& Lee, 2002). 
Procedimiento

El estudio fue aprobado por el Comité de Investigación del Centro de Rehabilitación e Integración Social (CRIS) del Sistema DIF del Estado de Aguascalientes.

\section{Evaluación pre test}

Evaluación de las características físicas de los puntos gatillo (PsG).

Durante el reclutamiento, mediante palpación (Sciottia, et al., 2001), un médico especializado adscrito al centro en donde se realizó la investigación determinaba el tamaño del nódulo blando y la banda tensa, así como la intensidad de dolor que le paciente percibía cuando se le presionaba el nódulo blando. Esta presión se realizaba con el pulgar de la mano derecha con una fuerza aproximada de 4 kg., según lo refiere Simons (2004). La palpación se realizaba con la paciente recostada boca abajo sobre una cama de auscultación. Todas las mediciones fueron consignadas por el especialista en una escala de calificación numérica (ECN). Esta evaluación confirmaba la presencia de SDM.

Evaluación de la intensidad percibida de dolor.

Durante la primera sesión se le pedía a la participante que anotara con un bolígrafo en una ECN cual había sido en promedio la intensidad percibida de dolor durante la semana inmediata pasada.

Evaluación de los niveles de actividad autonómica.

Una vez puntuada la ECN, se colocaban los sensores de temperatura en la zona de las manos que se encuentra entre los dedos índice y pulgar, y los electrodos en los músculos trapecios superiores. Se consideraba un tiempo de adaptación de cinco minutos durante los cuales se explicaban las evaluaciones que se realizarían durante la sesión (Perfil psicofisiológico e inventarios de ansiedad y depresión de Beck) para luego comenzar a tomar los registros propios del primero, el cual consistió en cuatro condiciones de dos minutos cada una, durante las cuales se registra la actividad de la señal elegida cada quince segundos a saber: 1 ojos abiertos. Se instruía a la paciente para permanecer sentada con sus ojos abiertos; 2 ojos cerrados. Se le pedía permanecer con sus ojos cerrados sin hacer otra cosa; 3 condición de estrés. Recordar con ojos cerrados un episodio de dolor reciente e intenso; y 4 desarrollar una respuesta de relajación natural, para ello debía cerrar los ojos y concentrarse en relajarse como supiera hacerlo hasta que se le indicara que el tiempo había finalizado (ver Ruvalcaba, 2011; Ruvalcaba \& Domínguez, 2009a).

Evaluación de los niveles de ansiedad y depresión.

Finalizado el perfil psicofisiológico y una vez retirados los sensores se entregaba a la paciente los inventarios de ansiedad y depresión de Beck (Jurado, et al., 1998; Robles, et al., 2001) y se le explicaba la forma de llenarlos. Después se le pedía que 
los respondiera. El tiempo promedio de aplicación fue de 20 minutos para los dos instrumentos.

Intervención

\section{Entrenamiento en relajación}

A partir de la segunda sesión las participantes del grupo A recibieron un entrenamiento de 10 sesiones de una hora, impartidas de manera individual una vez por semana bajo el siguiente esquema:

Las cuatro primeras sesiones la relajación era guiada por el IP con el objetivo de entrenar la respiración diafragmática. Las siguientes tres sesiones las instrucciones estaban diseñadas para que la paciente relajara los músculos trapecios superiores. Durante las tres sesiones restantes la participante practicaba la relajación muscular sin apoyo del IP. Durante las primeras siete sesiones se empleaban diez minutos de relajación guiada, un descanso de cinco minutos (aquí la paciente comentaba sus experiencias, aprendizajes y dificultades); para enseguida relajarse por sí misma durante diez minutos utilizando como apoyo retroalimentación auditiva.

Las últimas tres sesiones las pacientes controlaban la actividad de sus músculos trapecios únicamente mediante retroalimentación visual, para ello se relajaba durante cinco minutos por dos de descanso durante tres series.

Todas las pacientes en este grupo recibieron un disco compacto con instrucciones de relajación para que lo escucharan diariamente en casa (Ruvalcaba, 2011; Ruvalcaba \& Domínguez, 2009a).

\section{Pláticas para la salud}

Las participantes del grupo B recibieron 10 sesiones de charlas para la salud, las cuales y para mantener constantes las condiciones experimentales entre ambos grupos fueron conducidas por el IP, duraban una hora y eran impartidas una vez por semana de manera individual en el mismo consultorio en el que se recibía a las participantes del grupo A.

Los temas de las sesiones se programaron de la siguiente manera: en la sesión 1 "El dolor miofascial en espalda baja", en la 2 "Beneficio de la fisioterapia", 3 y 4 "Educación postural", en la 5 "Descanso después del trabajo", 6 "Ejercicios de fortalecimiento y estiramiento músculos de la espalda", 7 "Ejercicios de fortalecimiento y estiramiento músculos del abdomen y piernas", 8 "Nutrición", 9 "Adherencia terapéutica", 10 "Panorama general del dolor miofascial y su rehabilitación". Al finalizar cada sesión se entregaba a las participantes un resumen del tema.

\section{Evaluación postest}

Esta sesión se desarrolló igual que la evaluación pretest descrita anteriormente, a excepción de la evaluación de los PsG la cual fue realizada hasta el final, en el consultorio del médico especialista. El tiempo entre el pre y post test fue de tres meses 
Procedimiento estadístico

Se utilizó la prueba de rangos señalados y pares igualados de Wilcoxon para evaluar la influencia de la intervención en las variables estudiadas y un coeficiente de correlación $\eta^{2}$ con el objetivo de evaluar la magnitud de la relación entre el tratamiento y los cambios registrados en las variables. Esta prueba fue utilizada debido a que no está sujeta a la influencia del tamaño de la muestra (Kerlinger \& Lee, 2002). Todas se realizaron en el paquete estadístico SPSS versión 10 con un alfa de 0.05 .

Resultados

Descripción de la muestra

La edad promedio en ambos grupos fue de 46 años, ( $D S \pm 6.4$ para el grupo A y \pm 5.4 para el B). Todas las pacientes estaban diagnosticadas con dolor en espalda baja por contractura de masas musculares, desempeñaban trabajo administrativo y tenían en promedio tres años de haber presentado el primer episodio de dolor (DS \pm 1.1 años grupo $\mathrm{A} y \pm .72$ grupo B). El flujo de participantes se presenta en la figura 1 .
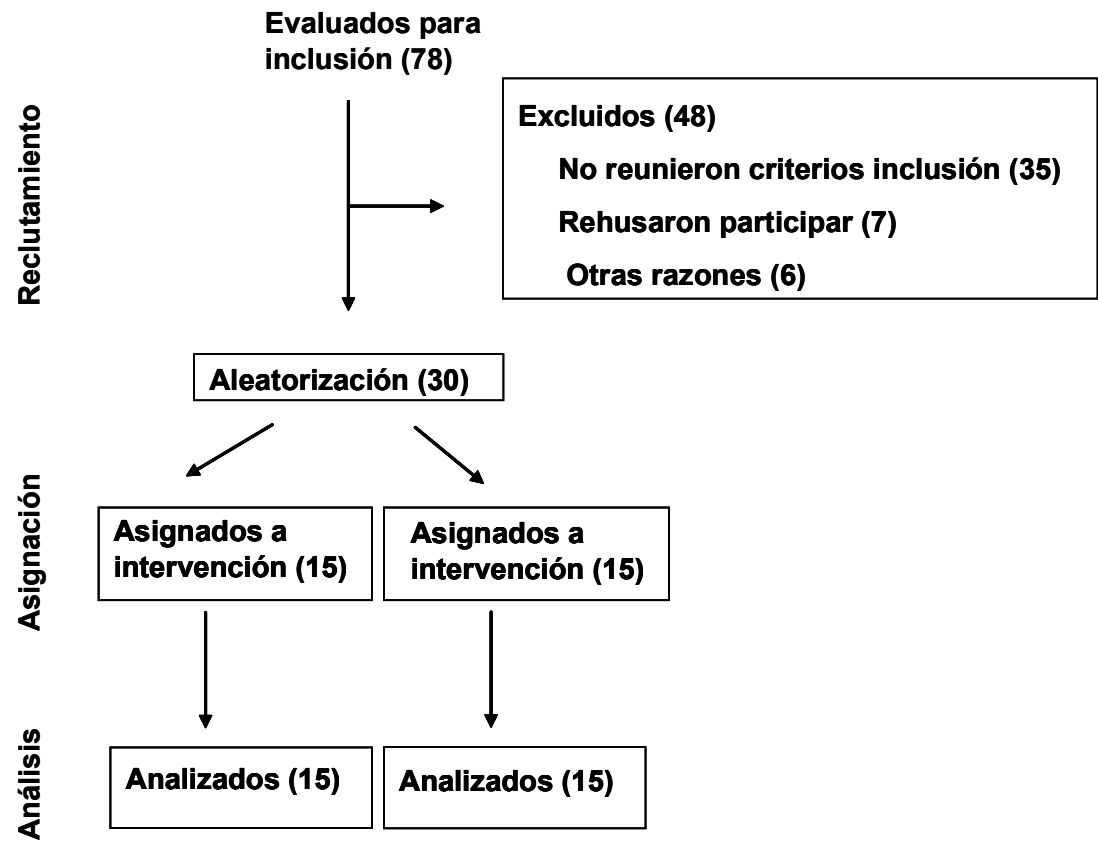

Figura 1. Flujo de participantes 
Valores por grupo en cada variable antes y después del tratamiento

En la tabla 2 se presentan los valores promedio de las diferentes variables en cada grupo antes y después de la intervención. Los valores pretest fueron similares en ambos grupos. Al final de la intervención los valores fueron significativamente diferentes $(\alpha=0.05)$, de tal manera que el grupo A mostró decrementos en todas las variables, no así el grupo B que se mantuvo en los mismos valores o con ligeros incrementos.

Tabla 2.

Valores por grupo antes y después del tratamiento

\begin{tabular}{|c|c|c|c|c|c|c|c|c|}
\hline \multirow{3}{*}{$\begin{array}{l}\text { VARIABLE } \\
\text { Temperatura }\end{array}$} & \multicolumn{4}{|c|}{ PRETEST } & \multicolumn{4}{|c|}{ POSTEST } \\
\hline & \multicolumn{2}{|c|}{ ENTRENAMIENTO } & \multicolumn{2}{|c|}{ CHARLAS } & \multicolumn{2}{|c|}{ ENTRENAMIENTO } & \multicolumn{2}{|c|}{ CHARLAS } \\
\hline & 3.02 & $( \pm 1.84)$ & 93.21 & $( \pm 1.45)$ & 95.46 & $( \pm 0.88)$ & 93.54 & $( \pm 0.99)$ \\
\hline EMGs & 3.02 & $( \pm 0.64)$ & 3.32 & $( \pm 0.69)$ & 2.05 & $( \pm 0.37)$ & 3.93 & $( \pm 0.46)$ \\
\hline Ansiedad & 21 & $( \pm 8.72)$ & 19.6 & $( \pm 8.17)$ & 7.13 & $( \pm 3.92)$ & 21.53 & $( \pm 7.77)$ \\
\hline Depresión & 15.93 & $( \pm 5.3)$ & 20.06 & $( \pm 5.32)$ & 7.33 & $( \pm 4.48)$ & 21.26 & $( \pm 8.11)$ \\
\hline Banda Tensa & 1.27 & $( \pm 1.84)$ & 2.07 & $( \pm 1.84)$ & 0.72 & $( \pm 0.54)$ & 2.34 & $( \pm 0.85)$ \\
\hline Nódulo Blando & 1.39 & $( \pm 1.00)$ & 2.00 & $( \pm 1.00)$ & 0.78 & $( \pm 0.15)$ & 2.36 & $( \pm 0.81)$ \\
\hline Dolor Presión & 2.28 & $( \pm 1.52)$ & 3.13 & $( \pm 1.52)$ & 1.01 & $( \pm 0.67)$ & 3.42 & $( \pm 0.97)$ \\
\hline Intensidad Dolor & 5 & $( \pm 1.46)$ & 5.46 & $( \pm 1.46)$ & 1.46 & $( \pm 1.55)$ & 6.53 & $( \pm 1.68)$ \\
\hline
\end{tabular}

Efectos de la intervención en los niveles de actividad autonómica

Temperatura

La media del grupo A antes del entrenamiento fue de $93.32{ }^{\circ} \mathrm{F}$ mientras que en la evaluación posterior se incrementó a 95.46. Este incremento fue estadísticamente significativo $(t=2.37, p<0.05)$. El grupo B no mostró cambios significativos $(t=$ $0.76, p<0.05)$. ver Tabla 1 y Figura 2.

Electromiografía de superficie (EMGs)

En la Tabla 1 y Figura 2. Puede observarse que el grupo A presentó una reducción significativa en sus registros EMGs pasando de una media de $3.02 \mu \mathrm{V}$, a una de 2.05 $\mu \mathrm{V}$ en la segunda evaluación $(t=4.7, \mathrm{p}<0.05)$. El grupo $\mathrm{B}$ mostró incrementos, pasando de $3.32 \mu \mathrm{V}$ a $3.93 \mu \mathrm{V}(t=1.6, p<0.05)$. 

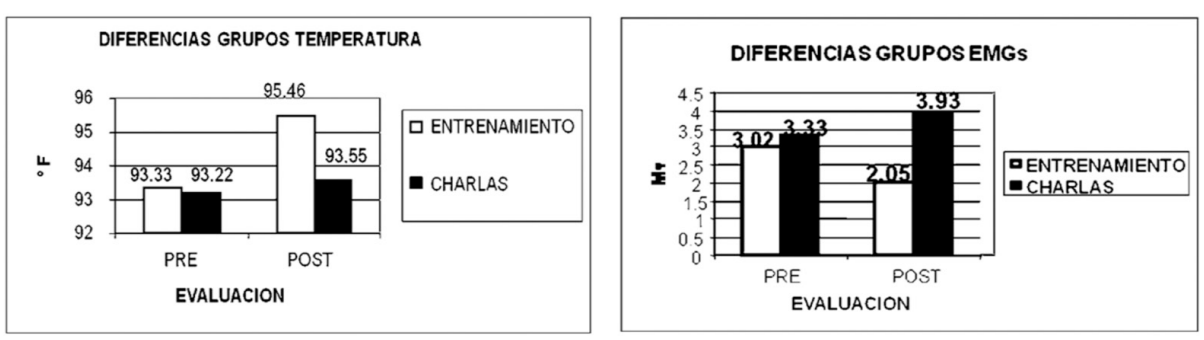

Figura 2. Diferencias en actividad autonómica (temperatura y EMGs) entre e intra grupos.

Nota: La temperatura está en grados Fahrenheit y la EMGs en micro volts $(\mu \mathrm{V})$. Se espera que la temperatura se incremente y la EMGs decremente. Los valores de la ansiedad y la depresión corresponden a puntajes crudos. La banda, el nódulo, el dolor a la presión y la intensidad de dolor corresponden a valores en una ECN donde 0 es igual a "nada" y 10 "el máximo grado de la presencia de la variable". Los valores entre paréntesis representan la desviación estándar.

\section{Efectos en el Estado Cognitivo Emocional}

\section{Ansiedad}

El grupo A, después del entrenamiento mostró decrementos significativos en los puntajes de ansiedad $(t=3, p<0.05)$, pasando de una media de 21 (nivel moderado) a 7.1 (nivel leve). En contraste (Figura 3), los puntajes promedio del grupo B permanecieron prácticamente igual: de 19.60 en la primera evaluación a 21.53 en la segunda, manteniéndose en un nivel moderado $(t=6, p<0.05)$

\section{Depresión}

El grupo A mostró una reducción significativa en sus puntajes después del entrenamiento ( $t=2, p<0.05$ ) pasando de 15.93 (depresión leve) a 7.33 (depresión mínima). El grupo B no mostró cambios significativos, pues la media del grupo en la primera evaluación fue de 20.06 y 21.26 en la segunda, en ambas el nivel de depresión fue moderado $(t=1, \mathrm{p}<0.05)$. Ver Figura 3.

\section{Efectos en las Características Físicas de los PsG}

Nódulo blando

Después de la intervención el grupo A pasó de una media de 1.39 puntos en la evaluación inicial, a .72 en la final $(t=1.7, p<0.05)$. El caso contrario ocurrió en el grupo B pues las pacientes presentaron aumento no significativo, pasando de 2.0 al inicio a 2.34 al final $(t=2, p<0.05)$, como se puede ver en la Figura 4. 


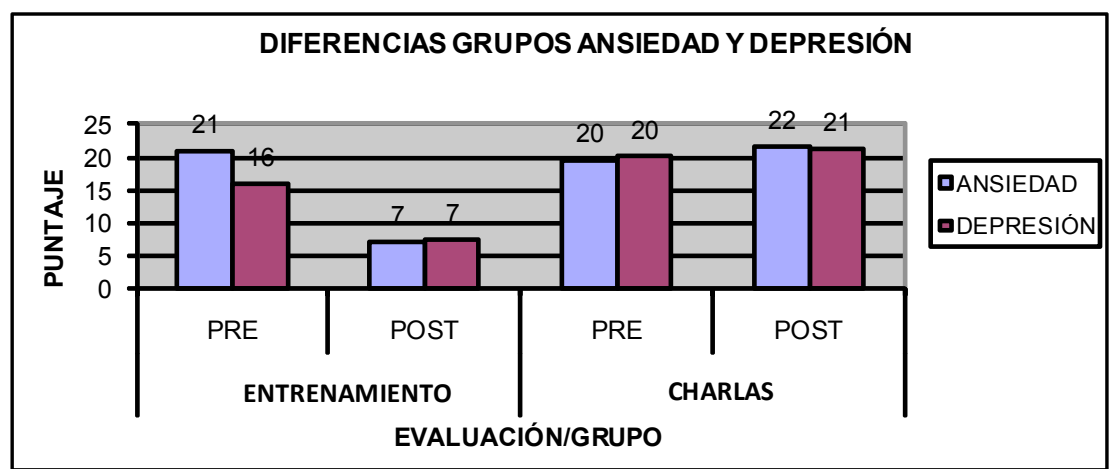

Figura 3. Diferencias en el estado cognitivo-emocional entre e intra grupos

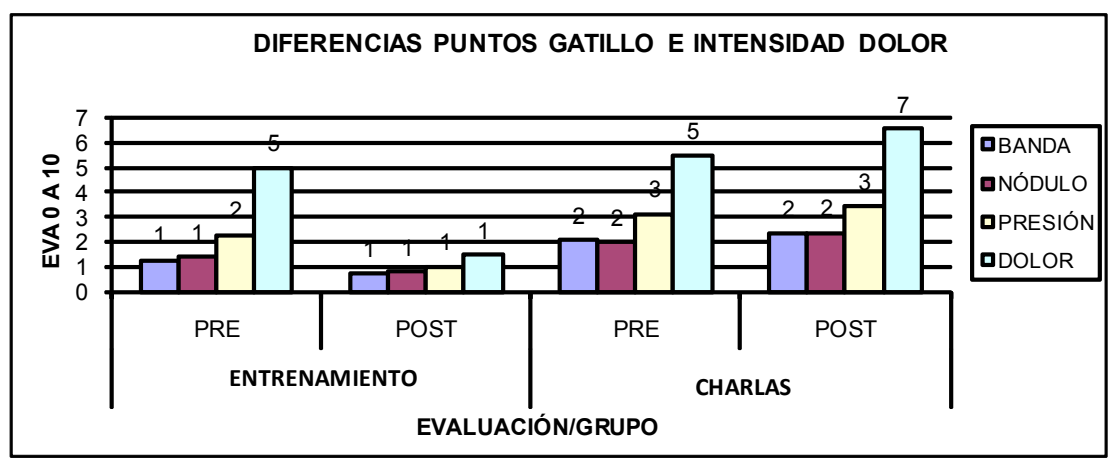

Figura 4. Diferencias entre e intra grupos características de los PsG ${ }^{\circ}$

\section{Banda tensa}

La diferencia entre las medias de esta variable antes y después de la intervención en el grupo A fue significativa. Antes del entrenamiento la media grupal fue de 1.27 y después del mismo disminuyó a $.78(t=1.3, p<0.05)$. Por su parte en el grupo $\mathrm{B}$ permanece casi igual, pasando de una media de 2.07 a una de 2.36 ( $\mathrm{t}=0.7, \mathrm{p}<$ 0.05). Ver Figura 4. 
Dolor a la Digitopresión

Las participantes en el grupo A manifestaron sentir un decremento significativo entre ambas mediciones $(t=3, p<0.05)$ pasando de una media de 2.28 a una de 1.01 después del mismo. El grupo $\mathrm{B}$ por su parte no mostró diferencias significativas $(\mathrm{t}=$ $0.5, \mathrm{p}<0.5)$.

\section{Intensidad Percibida de Dolor}

En la Figura 4 puede verse que en el grupo A la variable dolor muestra decrementos significativos entre una medición y otra: al inicio de la intervención la calificación promedio fue 5 y al final fue $1.46(t=3, p<0.05)$. En contraste el grupo B mostró incrementos significativos en los puntajes asignados a esta variable, pues al inicio el dolor se percibía con una intensidad de 5.46 y al final con una intensidad de 6.53 $(t=1.5, p<0.05)$.

Fuerza de la relación

Al aplicar un coeficiente de correlación curvilínea $\mathrm{\eta}^{2}$ se encontró una fuerte relación entre la modificación de la actividad autonómica y los cambios en las diferentes variables, esta relación fue significativa con un $\alpha=0.05$.

\section{Discusión}

En la TP se propone una estrecha relación entre las estrategias conductuales, emocionales y fisiológicas que integran las conductas adaptativas de los organismos y el funcionamiento autonómico que subyace a dichas estrategias y que la modificación en este último traerá modificaciones en las primeras.

El propósito de este estudio fue evaluar si los cambios en el funcionamiento autonómico de un grupo de pacientes con dolor miofascial en espalda baja se acompañaban por cambios en su estado cognitivo emocional, las características físicas de los PsG y la intensidad percibida de dolor y así reunir evidencia sobre si los supuestos anteriores aplicaban para este tipo de pacientes y de esta forma contribuir al entendimiento del desarrollo, permanencia y tratamiento de síndromes crónicos musculares.

Después de la intervención, el grupo que aprendió a modular su actividad autonómica presentó decrementos en los síntomas, principalmente en el estado cognitivo emocional y en la intensidad percibida de dolor. Mientras que el grupo control no. Respecto a las variables físicas, los participantes del primer grupo mostraron decrementos significativos en las características diagnósticas de los PsG, mientras que el segundo tendió al incremento de los síntomas.

La evidencia obtenida permite confirmar la hipótesis establecida y afirmar que, tal y como se plantea en la TP, la actividad autonómica juega un papel importante en el desarrollo de los síntomas físicos y psicológicos que acompañan al SDM. Así mismo, es muy posible que el funcionamiento adaptativo ante el dolor muscular agudo sea un factor a considerar en el establecimiento y permanencia del síndrome 
crónico, incluyendo los síntomas psicológicos que le acompañan y la conducta general de dolor-enfermedad.

El hecho de que las variables de naturaleza psicológica hayan presentado los cambios más grandes podría deberse directamente a un efecto de la intervención sobre el nivel de funcionamiento del sistema nervioso simpático. Ya en otras partes (Ruvalcaba \& Domínguez, 2009b; Turk, 2004) se ha documentado que los principales beneficios de las intervenciones cognitivo conductuales se relacionan con decrementos en la intensidad percibida de dolor y una mejoría en el estado de salud y emocional en general. Además ayudan a los pacientes a desarrollar pensamientos que les permiten sentirse capaces de enfrentar los episodios de dolor, tener menos pensamientos catastróficos sobre éste e incrementar la percepción de que pueden controlar la intensidad de los síntomas (Gatchel, Peng, Peters, Fuchs \& Turk, 2007). Todos estos factores cognitivos influyen en la disminución de la ansiedad y depresión asociada con los trastornos crónicos (Ruvalcaba \& Domínguez, 2009b), y con una disminución de la actividad del eje HPA, la cual es mediada por la actividad nerviosa simpática (Gaab, Rohleder, Nater \& Ehlert, 2005).

En este sentido las intervenciones encaminadas a mejorar el funcionamiento autonómico, como por ejemplo aquellas basadas en la retroalimentación biológica, ayudan a mejorar en los pacientes los aspectos emocionales, como la ansiedad y la depresión y a la vez a tener una mejor calidad de vida, una menor utilización del servicio médico, menor tiempo de recuperación y solicitar una menor cantidad de días por incapacidad (Ruvalcaba \& Domínguez, 2009b; Turk, 2004), tal y como sucedió en el presente estudio.

Respecto a los cambios que los pacientes registraron en las características físicas de los PsG, la revisión de la literatura permite determinar que pueden deberse también directamente a la modulación de la actividad autonómica y a los efectos de ésta tanto en el estado cognitivo emocional, como en la activación fisiológica relacionada con la actividad muscular. Ya otros científicos han mostrado cómo incrementos en la actividad parasimpática se relacionan con mejoría en los síntomas físicos y con disminuciones en la intensidad percibida de dolor y en los estados de ansiedad y depresión de los pacientes con SDM (Takamoto, et al.,2009; Zhang, Yue, Kimura \& Arendt-Nielsen, 2009) y aunque se acepta que la actividad simpática y parasimpática no son completamente antagónicas y que la diminución en una no supone necesariamente un incremento en la otra (Jänig, 2009), si puede decirse que las intervenciones basadas en la relajación y la retroalimentación biológica (como la realizada en el presente estudio) tienen el efecto de aumentar la actividad parasimpática y disminuir la simpática por lo que los resultados obtenidos sirven de base para aceptar que la actividad autonómica es un elemento que debe considerarse para comprender completamente los mecanismos que subyacen a la instalación, permanencia, desarrollo y tratamiento de los síndromes crónicos musculares.

Estos resultados tienen varias implicaciones para el campo de la medicina conductual. Primero, constituyen evidencia para visualizar el uso de la TP como un 
marco teórico que permita interpretar el desarrollo y permanencia del SDM desde una perspectiva evolutiva que facilite la integración de aquellos procesos psicológicos y neurobiológicos que intervienen en el establecimiento del estado crónico de enfermedad, en un conjunto de respuestas y reacciones adaptativas susceptibles de ser modificadas conscientemente a través del entrenamiento.

Segundo, se abre la posibilidad para considerar que los síndromes crónicos de dolor muscular se desarrollan y permanecen debido en gran parte a la utilización sostenida de estrategias adaptativas que son adecuadas para enfrentar el evento de dolor agudo, pero que no lo son para enfrentar la lesión crónica. Tercero, es posible la emergencia de un nuevo campo de estudio e intervención en el que la necesidad de comprender cómo las reacciones adaptativas y las estructuras nerviosas centrales que subyacen a ellas intervienen en la histopatogenia y las manifestaciones sintomáticas (tanto físicas como psicológicas) sea un imperativo que no puede ser ignorado

Es claro que los resultados y las conclusiones anteriores deben ser tomadas con cautela debido a las limitaciones que presentó el estudio como son: el número de participantes reducido y con características muy específicas (mujeres, con dolor miofascial en espalda baja, trabajadoras administrativas). Además no se realizó un seguimiento que permitiera comprobar si la modificación de la actividad autonómica y el decremento de los síntomas permanecen en el tiempo. Por otro lado se recomienda utilizar otras señales que complementen el panorama del funcionamiento nervioso y que tengan más estabilidad para su análisis y registro como son la Electrodermografía y la Variabilidad de la Frecuencia Cardiaca. También se recomiendan hacer estudios que incluyan un mayor número de pacientes con diferentes tipos de síndromes musculares o de dolor crónico.

\section{Conclusión}

En el sistema nervioso residen los procesos psicológicos y fisiológicos a través de los cuales el ser humano se adapta al medio ambiente, incluyendo aquellos relacionados con la salud y la enfermedad (Cacioppo \& Decety, 2009). El reto actual para los psicólogos dentro del área de la medicina conductual es definir adecuadamente aquellos constructos psicológicos que permitan una comprensión holística del ser humano, que contemplen la inseparable relación entre procesos psicológicos, fisiológicos/biológicos y sociales (Cacioppo \& Decety, 2009; Porges, 2006). Esto implica la consideración no solamente de las intrincadas redes neuronales que hacen posible la percepción y la conducta, sino también de aquellos procesos genéticos, moleculares y celulares que impactan directamente en la función neuroendócrina, el desarrollo de síndromes crónicos y la integración de estados de salud.

En el caso del dolor miofascial, la TP puede ser un adecuado marco de referencia teórica que permita contemplar, desde una perspectiva evolutiva, la regulación nerviosa, la actividad fisiológica y los principios neuroconductuales adaptativos como elementos importantes en el desarrollo del síndrome, y por supuesto, en la integración 
de intervenciones psicológicas basadas en la regulación cognitiva-emocional y el control volicional de la actividad autonómica. De esta forma la medicina conductual se vería enriquecida con una práctica basada en los procesos psicofisiológicos directamente asociados con las respuestas evolutivo-adaptativas que podrían estar ligadas al origen del SDM.

\section{Referencias}

Bistre, C.S. (2009). Impacto social y humano del dolor crónico. En C.S. Bistre (Ed.), Dolor: cuidados paliativos, diagnóstico y tratamiento (pp. 465-467), México, D.F.: Trillas.

Cacioppo, J.T., \& Decety, J. (2009). What are the brain mechanisms on which psychological processes are based? Perspectives on psychological science 4 (1), 10-18. Available via: http://dx.doi.org/10.1111/j.1745-6924.2009.01094.x

Chrousos, G.P. (1992). Regulation and dysregulation of the hipotalamic-pituitary-adrenal axis. Endocrinology and Metabolism Clinics of North America, 21, 833-858.

Chrousos, G.P., \& Gold P.W. (1992). The concepts of stress and stress system disorders. Journal of the American Medical Informatics Association: JAMIA 267, 1244-1252.

Gaab, J., Rohleder, N., Nater, U.M., \& Ehlert, U. (2005). Psychological determinants of the cortisol stress response: the role of anticipatory cognitive appraisal. PSyconeuroendocrinology. 30, 599-610. Available via: http://dx.doi.org/10.1016/ j.psyneuen.2005.02.001

Gatchel, R.J., Peng, Y.B., Peters, M.L., Fuchs, P.N., \& Turk, D.C. (2007). The biopsychosocial approach to chronic pain: Scientific advances and future directions. Psychological Bulletin. 13(4), 581-624. Available via: http://dx.doi.org/10.1037/ 0033-2909.133.4.581

Huggenin, K.L. (2004). Miofascial Trigger Points: The current evidence. Physical Therapy in Sport; 5, 2-12

Jänig, W. (2009). Autonomic nervous system and pain. En A.I., Basbaum \& C.,Bushnell (Eds.), Science of pain (pp. 194-221). Oxford: Elsevier Academic Press.

Jurado, S., Villegas, M.E., Méndez, L., Rodríguez, F., Loperena, U.V., \& Varela, R. (1998) La estandarización del Inventario de Depresión de Beck para los residentes de la ciudad de México. Salud Mental; 5, (21), 26-31.

Kerlinger, F.N., \& Lee, H.B. (2002) Investigación del comportamiento. Métodos de investigación en ciencias sociales. México, D.F.: McGraw-Hill.

McQuay, H. (2005). Consensus on outcome measures for chronic pain trials [Editorial]. Pain; 113, 1-2.

Melzack R. (1999). Pain and Stress: A New Perspective. En R.J. Gatchel \& D.C. Turk (Eds.), Psychosocial Factors in Pain. (pp. 89-106). New York: The Guilford Press.

Myburgh C., Larsen A.H., \& Hartvigsen, J. (2008). A systematic, critical review of manual palpation for identifying miofascial trigger points: Evidence and clinical significance. Archives of Physical Medicine and Rehabilitation,31, 1169-1176. Available via: http://dx.doi.org/10.1016/j.apmr.2007.12.033 
Porges, S.W. (2001). The Polivagal theory: Phylogenetic substrates of a social nervous system. International Journal of Psychophysiology; 42, 123-146. Available via: http://dx.doi.org/10.1016/S0167-8760(01)00162-3

Porges, S.W. (2006). Asserting the role of biobehavioral sciences in translational research: The behavioral neurobiology revolution. Development and Psychopathology, 18, 923-933. Available via: http://dx.doi.org/10.1017/S0954579406060457

Porges, S.W. (2007). A phylogenetic journey through the vague and ambiguous Xth craneal nerve: A commentary on contemporary Herat rate variability research. Biological Psychology, 74, 301-307. Available via: http://dx.doi.org/10.1016/ j.biopsycho.2006.08.007

Porges, S.W., Domínguez, T.B., Rangel, G.E., \& Cruz, M.A. (2005). La teoría polivagal, entendiendo los mecanismos del estrés postraumático. CONACYT Proyecto MO 299, Mexico. DF.: Universidad Nacional Autónoma de México.

Punnett, L., \& Wegman, D. H. (2004) Work-related musculoskeletal disorders: the epidemiologic evidence and the database. Journal of Electromiography and Kinesiology 14, 13 - 23.

Robles, R., Varela, R., Jurado, S., \& Páez, F. (2001). Versión mexicana del Inventario de Ansiedad de Beck: Propiedades psicométricas. Revista Mexicana de Psicología; 18(2), 211-218.

Ruvalcaba, P.G., (2011). Efectos psicológicos y físicos de la modulación autonómica en pacientes con dolor miofascial. Disertación doctoral no publicada, Universidad Nacional Autónoma de México, México, D.F.

Ruvalcaba, P.G., \& Domínguez, T.B. (2009a). Uso de la retroalimentación biológica en el dolor crónico. En C.S. Bistre (Ed.) Dolor: Cuidados Paliativos, Diagnóstico y Tratamiento (pp. 366-378), México: Ed. Trillas.

Ruvalcaba, P.G. \& Domínguez, T.B. (2009b). La terapia psicológica del dolor crónico. Psicología y Salud, 19 (2), 247-252.

Sapolsky, R. (2001). Depression antidepressants and the shrinking hippocampus. PNAS; 98,12320-12322. Available via: http://dx.doi.org/10.1073/pnas.231475998

Sapolsky, R. (2003). El control del estrés. Scientific American Lationoamerica; 16, 60-68.

Schulkin, J. (2003). Rethinking homeostasis: allostatic regulation in physiology and pathophysiology. Massachusetts: Massachusetts Institute of Technology Press.

Sciottia, V.M., Mittaka. L.V., DiMarcoa, L., Forda, L.M., Plezberta, J., \& Santipadria, E. (2001). Clinical precision of myofascial trigger point location in the trapezius muscle. Pain; 93, 259-266.

Simons, G.D. (2004). Review of enigmatic MTrPs as a common cause of enigmatic musculoskeletal pain and dysfunction. Journal of Electromyography and Kinesiology, 14, 95-107. Available via: http://dx.doi.org/10.1016/j.jelekin.2003.09.018

Staud, R. (2009). Fibromyalgia. En: A.I. Bausbaum \& C. Bushnell (Eds.), Science of pain (pp. 775-780). San Diego CA: Academic Press.

Tait, C.R. (1999). Psychosocial factors in pain: Evaluation of treatment effectiveness 
in patients with intractable pain. Measures and methods. En: R.J. Gatchel \& Turk, D.C. (Eds.), Psychosocial factors in pain: Critical perspectives (457-493) New York, USA: Guilford Press.

Takamoto, K., Sakai, S., Hori, E., Urakawa, S., Umeneo, K., Ono, T., \& Nishijo, H. (2009) Compression on trigger points in the leg muscle increases parasympathetic nervous activity based on hearth rate variability. Journal of Physiological Sciences; 59, 191-197. Available via: http://dx.doi.org/10.1007/s12576-009-0025-y

Turk, D.C. (2004). Understanding pain sufferers: the role of cognitive processes. The Spine Journal; Editorial, 4, 1-7. Available via: http://dx.doi.org/10.1016/S15299430(03)00068-8

Zhang, Y., Ge, H., Yue, S., Kimura, Y., \& Arendt-Nielsen, L. (2009). Attenuated skin blood flow response to nociceptive stimulation of latent myofascial trigger points. Archives of Physical Medicine and Rehabilitation; 90 (2), 325-332. Available via: http://dx.doi.org/10.1016/j.apmr.2008.06.037 